\title{
A Monte Carlo Simulation Model for Evaluating the Effectiveness of Interventions along the 'Diabesity' Pathway
}

\author{
Anita Lynam \\ Exeter Medical School \\ University of Exeter \\ Exeter, United Kingdom \\ ag616@exeter.ac.uk
}

\author{
Doris A. Behrens \\ Aneurin Bevan Continuous Improvement \\ Aneurin Bevan University Health Board \\ Caerleon, United Kingdom \\ doris.behrens@wales.nhs.uk
}

\author{
Enzo M. Battista-Dowds \\ Adult Weight Management Service \\ Aneurin Bevan University Health Board \\ Caerleon, United Kingdom \\ enzo.dibattista@wales.nhs.uk
}

\author{
Daniel Gartner \\ School of Mathematics \\ Cardiff University \\ Cardiff, United Kingdom \\ gartnerd@ cardiff.ac.uk
}

\author{
Paul Harper \\ School of Mathematics \\ Cardiff University \\ Cardiff, United Kingdom \\ harper@ cardiff.ac.uk
}

\begin{abstract}
The prevalence of obesity has increased worldwide in the past 50 years, reaching pandemic levels (Blüher, 2019). In this paper, we report on the development of an intervention evaluation model and risk assessment tool that has been developed for an Adult Weight Management Service (AWMS) within the U.K.'s National Health Service (NHS). The tool uses Monte Carlo simulation to predict the progress of morbidity and mortality in individual AWMS patients for up to 25 years, with and without intervention. Running the tool on a sample of AWMS patients with known weight loss outcomes indicates that interventions can be evaluated in terms of risk assessment of developing obesityrelated health conditions such as diabetes.
\end{abstract}

Index Terms-Monte Carlo Methods, Prognostics and Health

\section{INTRODUCTION}

Obesity is an "abnormal or excessive fat accumulation that may impair health" [9] and forecasting models predict more than $51 \%$ of the U.S. population as obese through 2030 [4]. There is some debate over the most appropriate measure for identifying obesity. The most commonly used measure in the U.K. and the one used in this paper is Body Mass Index (BMI); adults with a BMI greater or equal to 30 are classified as obese. BMI is a straightforward method of identifying obesity; however, its validity and usefulness are questionable. For example, no adjustment is made for factors such as sex, muscle mass, ethnic origin and adipose tissue distribution, making it difficult to identify those patients most at risk of developing obesity-related health issues based only on their BMI.

There are at least 30 chronic health conditions associated with adult obesity including: coronary heart disease (CHD), stroke, type II diabetes, cancers (oesophagus, breast, endometrium, colon, kidney, pancreas, thyroid, gallbladder), hy-

The authors greatly appreciate the financial support received from the Bevan Commission's Bevan Exemplar scheme as well as the Health and Care Research Wales' Pathway to Portfolio scheme. pertension (high blood pressure), dyslipidaemia (high cholesterol or high levels of triglycerides), gastrointestinal and liver disease, musculoskeletal problems (osteoarthritis and lower back pain), gynaecological and obstetric complications, respiratory problems (asthma and sleep apnoea) and psychological and emotional problems (depression, anxiety and low selfesteem) [1]. In this project only health conditions associated with both obesity and type II diabetes are considered: prediabetes, type II diabetes, stroke, CHD, retinopathy, neuropathy and nephropathy.

Patients with pre-diabetes have a high risk of developing diabetes due to their elevated blood glucose levels. All patients with diabetes will have progressed from pre-diabetes, although it often goes undiagnosed, and the duration in pre-diabetes is highly variable.

Weight loss achieved through lifestyle interventions can prevent or delay the progression from pre-diabetes to diabetes [6]. Real risk reduction, however, is achieved by patients returning to normal glucose regulation (NGR) and participation in lifestyle interventions alone doubles the likelihood of this [7].

In this paper, we report on the development of a Monte Carlo simulation model which takes several parameters into accounts such as BMI and patients' gender to support an Adult Weight Management Service (AWMS) in Wales, U.K. The model can report the likelihood of obesity-related risk factors such as diabetes and helps clinicians in NHS Wales to help patients understand their individual risk for developing obesity-related conditions.

\section{Methodology}

Monte Carlo simulation is a type of computer simulation that relies on repeated random sampling and statistical analysis to compute the results [8]. We use this approach to be able to 
incorporate empirical evidence from the medical literature in our analysis of whether patients are likely to develop obesityrelated conditions.

Our model consists of four core states: (1) normal glucose regulation (2) impaired glucose regulation (= pre-diabetes), (3) type II diabetes and (4) death. For the type II diabetes state, we distinguish, moreover, between five different health states that generate different levels of treatment cost for the NHS. Random number generation determines whether the patient moves between these core states and "develops" certain conditions. Annual transition probabilities are functions of both patient- and dwell-time-related attributes.

Our Monte Carlo simulation model was built in Microsoft Excel, and the state space and trajectories between states were coded in Visual Basic for Applications. The rationale to use MS Excel was because the tool should be used and maintained by NHS staff. It incorporates an individual-level simulation model that tracks the progression of morbidity (and mortality) for an AWMS patient for up to 25 years following intervention such as keeping weight constant or weight losses, specifically the onset of stroke, coronary heart disease (CHD), pre-diabetes, type II diabetes, retinopathy, neuropathy and nephropathy.

The tool has a particular feature to offer the evaluation of two different scenarios: non-intervention and intervention. In line with the medical literature, we assume for the nonintervention scenario that a patient's initial weight increases by one kilogram per year until the age of 70 (where the weight gain starts to level off). For the intervention scenario, we assume that the initial weight loss of usually up to $2.5 \mathrm{~kg}$ during the first year is sustained for another year with weight then increasing annually by one kilogram until the initial weight is regained (with then keeping weight constant). Our algorithm repeats this 1,000 times per run to reduce sampling variability errors. If weight loss following intervention is not known, our approach can be run using conservative (literaturebased) estimates for each intervention.

For each scenario, the mean total of the 25-year cost estimates over the 1,000 simulations is calculated. It is the difference between these two estimates that determines if the intervention reduces total treatment cost for the NHS. A comparison of the cumulative cost estimates over the 25 years provides an estimate for the number of years required to recoup the intervention costs. The cost of intervention is defined as the direct salary costs attributed to the delivery of the intervention; annual healthcare costs for each intervention were obtained from published literature.

We incorporated Copley's [3] diabetes model into our tool, which applies to ages 18 and above, and BMI above 22 . This is a model of an increase in prevalence by BMI, rather than incidence. However, given that diabetes is not an acute condition, this is felt to be a reasonable approximation, and is conservative given the increase in mortality associated with diabetes.

\section{Case Study Results}

To evaluate the time-limited AWMS interventions, our model was run 44 times for "average" AWMS patients (with and without diabetes). For their characteristics, see Table I. The planning horizon was set to $T=25$ years.

TABLE I

"AVERAGE PATIENTS" CHARACTERISTICS

\begin{tabular}{ccccc}
\hline Gender & Age & Start weight $(\mathrm{kg})$ & Height $(\mathrm{cm})$ & BMI \\
\hline Male & 52 & 123.6 & 168 & 43.8 \\
Female & 48 & 121.0 & 166 & 43.9 \\
\hline
\end{tabular}

For diabetic patients (type II), none of the AWMS interventions showed a statistically significant cost reduction for the NHS $(p<0.001)$. For non-diabetic patients, however, a marginal weight loss delayed the onset of the disease long enough to recoup intervention cost; all types of AWMS interventions paid off for the NHS after 5 to 11 years for men and 6 to 12 years for women.

\section{CONCLUSION}

In this paper, we report on the development of an intervention evaluation tool tailored to meet the requirements of an Adult Weight Management Service (AWMS). The tool is a simplistic representation of obesity; onset of obesity- and diabetes-related conditions which depend only on a limited number of factors including age, BMI and sex.

The tool can be used to demonstrate the effectiveness of AWMS interventions, predict required levels of weight loss and quantify, for example, mortality, morbidity and the expected cost of obesity in the future. Future work may include quality of life measures such as Quality Adjusted Life Years (QUALYs).

\section{REFERENCES}

[1] Aronne LJ. Classification of obesity and assessment of obesity-related health risks. Obesity Research, 10(S12):105S-115S, Dec 2002.

[2] Blüher M. Obesity: Global epidemiology and pathogenesis. Nature Reviews Endocrinology, 15(5):288-298, May 2019.

[3] Copley V. User Guide: Weight Management Economic Assessment Tool, Version 2. Oxford: Public Health England, Obesity Risk Factors Intelligence, 2016.

[4] Finkelstein EA, Khavjou OA, Thompson H, Trogdon JG, Pan L, Sherry B, Dietz W. Obesity and severe obesity forecasts through 2030. American Journal of Preventive Medicine, 42(6):563-570, Jun 2012.

[5] Kopelman PG. Obesity as a medical problem. Nature, 404(6778):635643, Apr 2000.

[6] Norris SL, Zhang X, Avenell A, Gregg E, Bowman B, Schmid CH, Lau J. Long-term effectiveness of weight-loss interventions in adults with pre-diabetes: a review. American Journal of Preventive Medicine, 28(1):126-139, Jan 2005.

[7] Perreault L, Kahn SE, Christophi CA, Knowler WC, Hamman RF. Diabetes Prevention Program Research Group. Regression from prediabetes to normal glucose regulation in the diabetes prevention program. Diabetes Care, 32(9):1583-1588, Sep 2009.

[8] Raychaudhuri S. Introduction to Monte Carlo simulation. In WSC'08: IEEE Proceedings of the 40th Conference on Winter Simulation, pp. 91-100, Dec 2008.

[9] World Health Organisation (2016). 